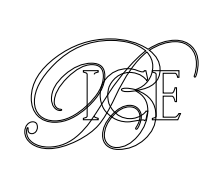

\title{
EL IMPACTO DE LA PANDEMIA EN EL MERCADO DE ALOJAMIENTOS TURÍSTICOS EN ESPAÑA: HOTELES VERSUS ALOJAMIENTOS P2P
}

El objetivo de este artículo es analizar el impacto que ha tenido la pandemia de la COVID-19 en el mercado de alojamientos turísticos distinguiendo los hoteles de los alojamientos p2p, tomando como referencia el caso español. Los datos provienen, por un lado, de la Encuesta de ocupación hotelera, y, por otro, de la Medición del número de viviendas turísticas en España y su capacidad y de la Estimación de la ocupación en alojamientos turísticos a partir de datos de plataformas digitales que, recientemente, ha publicado el INE. Por el lado de la oferta, durante la pandemia, los hoteles cerraron temporalmente, mientras que todo apunta a que en el mercado $\mathrm{p} 2 \mathrm{p}$ muchos propietarios de viviendas decidieron abandonarlo definitivamente. En cuanto a la demanda, el impacto negativo de la pandemia fue, en términos de pernoctaciones, relativamente menor en el mercado de alojamientos p2p que en el de los hoteles.

Palabras clave: COVID-19, viviendas turísticas, pernoctaciones.

Clasificación JEL: D11, L83.

\section{Introducción}

Una de las características más sobresalientes que está teniendo la descomunal crisis económica que se desencadenó a principios de 2020, como consecuencia de la COVID-19, ha sido la enorme diversidad de su impacto entre las distintas actividades productivas (Banco de España, 2021). En efecto, las medidas implementadas para tratar de contener la expansión del virus han impactado, de manera

* Universidad de Málaga.

Versión de enero de 2022.

https://doi.org/10.32796/bice.2022.3143.7345 especial, en aquellas actividades que requieren una elevada interacción personal, entre las que destaca el turismo. De hecho, debido a la variedad de empresas que, de una u otra forma, contribuyen a ofrecer un determinado producto turístico y que operan en diferentes mercados, la crisis ha afectado de forma desigual a lo que, de una manera genérica, denominamos sector turístico (UNWTO, 2019). Incluso para mercados concretos cualquier generalización resulta arriesgada. Por ejemplo, si pensamos en el mercado de alojamientos turísticos, es necesario diferenciar, al menos, los hoteles y otros alojamientos convencionales de las $D$ 
viviendas privadas que se comercializan a través de Internet gracias a plataformas como Airbnb.

El crecimiento del mercado peer-to-peer (p2p) de alojamientos turísticos ha supuesto una innovación disruptiva (Guttentag, 2015) que en las dos últimas décadas ha experimentado un crecimiento exponencial proporcionando una alternativa a los alojamientos turísticos tradicionales. En este sentido, las cifras que proporciona Airbnb (2022) son muy elocuentes: 5,6 millones de anuncios activos en más de 100.000 localidades de prácticamente todos los países del mundo. A nivel global, ha superado la capacidad de alojamiento de las cinco principales cadenas hoteleras del mundo (Hartmans, 2017) y, por ejemplo, en algunas ciudades españolas la oferta de Airbnb es superior al total de la planta hotelera (Jiménez et al., 2021). Las viviendas disponibles para su alquiler a corto plazo a través del mercado $\mathrm{p} 2 \mathrm{p}$ representan, por tanto, una parte sustancial del mercado de alojamientos turísticos y, en la medida que posee sus propias características (Benítez-Aurioles, 2020a), hace aconsejable un tratamiento diferenciado. Precisamente el objetivo de este artículo es analizar el impacto que ha tenido la pandemia de la COVID-19 en el mercado de alojamientos turísticos, distinguiendo los hoteles de los alojamientos p2p, para verificar la presencia de dinámicas diferenciadas, tomando como referencia el caso español.

\section{Repaso de la literatura}

En poco tiempo la literatura que relaciona la COVID-19 con el turismo ha experimentado un crecimiento considerable con numerosas aportaciones desde distintos ámbitos académicos
(Yang et al., 2021). Así, se han estudiado los efectos del incremento de la percepción del riesgo tanto para los turistas (Bae y Chang, 2021) como para la población residente (Tse y Tung, 2021), las motivaciones para viajar al extranjero (Rachmawati y Shishido, 2020), el cumplimiento de las medidas de seguridad en los hoteles (Hu et al., 2021), las propuestas para la resiliencia (Sharma et al., 2021), las respuestas de los Gobiernos (Collins-Kreiner y Ram, 2021) y de la propia industria hotelera (Lai y Wong, 2020), los esfuerzos para garantizar la sostenibilidad del turismo (HigginsDesbiolles, 2021) o las soluciones tecnológicas a los problemas que plantea la pandemia (Gretzel et al., 2020).

Respecto a los estudios de impacto, al margen de algunas aportaciones cualitativas (Bausch et al., 2021), se han realizado estimaciones cuantitativas mucho más numerosas para la industria turística convencional (Duro et al., 2021; Farzanegan et al., 2021; Huang et al., 2020; Kaczmarek et al., 2021; Khan et al., 2021; Lee y Chen, 2020; Qiu et al., 2020; Sharma y Nicolau, 2020; Škare et al., 2021; Tran et al., 2020) que para el mercado p2p de alojamientos turísticos (Benítez-Aurioles, 2021a; Gyódi, 2021; Jang y Kim, 2022; Llaneza Hesse y Raya Vilchez, 2021). Sin embargo, a lo que se nos alcanza, aún no se dispone de un análisis comparativo de la cuantificación de los efectos que ha provocado la pandemia en los hoteles y en los alojamientos p2p. Así, por ejemplo, en el libro de Nhamo et al. (2020) se incluye un capítulo titulado «Impacts and Implications of COVID-19 on the Global Hotel Industry and Airbnb» pero sin aportar datos que permitan una comparación sobre bases homogéneas.

No obstante, en el periodo prepandémico, un tema que atrajo la atención fue el $\triangleright$ 
impacto que estaba causando la expansión del mercado $\mathrm{p} 2 \mathrm{p}$ de alojamientos turísticos sobre el desempeño de la industria hotelera tradicional. En este contexto, aunque algunos estudios no detectaron una influencia clara (Blal et al., 2018; Choi et al., 2015; Heo et al., 2019; Strømmen-Bakhtiar y Vinogradov, 2019), otros han encontrado evidencia de que la actividad desarrollada por Airbnb afecta negativamente a la ocupación o rentabilidad de los hoteles (Benítez-Aurioles, 2019; Dogru, Hanks et al., 2020; Dogru, Mody et al., 2020; Xie y Kwok, 2017; Zervas et al., 2017).

Sobre la base de los estudios previos, la pandemia plantea un escenario nuevo para valorar las interacciones entre el mercado hotelero y el de alojamientos p2p. En particular, la preocupación por las condiciones sanitarias y la necesidad de mantener la distancia física ha podido afectar a la elección de los alojamientos que hacen los turistas. De hecho, igual que parece evidente que la demanda de habitaciones o pisos compartidos ha sido menor, también es posible que, en igualdad de condiciones, se prefieran los apartamentos completos que se ofrecen a través de Airbnb a las habitaciones de hotel (Bresciani et al., 2021). Surge, por tanto, la pertinencia de comprobar si, en efecto, durante la pandemia se han producido dinámicas diferentes en los mercados hoteleros respecto al de alojamientos p2p. La oportunidad de este estudio se presenta ante la reciente publicación de información estadística que permite un análisis comparativo con datos fiables.

\section{Datos}

La información que proporciona el Instituto Nacional de Estadística (INE) sobre la evolución de las variables básicas que afectan a los alojamientos hoteleros es bastante completa y tiene una cierta tradición. En particular, la Encuesta de ocupación hotelera aporta información mensual sobre la demanda y la oferta del sector hotelero con un aceptable grado de desagregación (INE, 2019). La unidad estadística es el establecimiento hotelero ya sea hotel, apartahotel, motel, hostal, pensión, fonda o casa de huéspedes. Los datos provienen de una encuesta que se realiza de manera exhaustiva en todas las provincias, excepto para algunos establecimientos de menor categoría, a partir de la cual el INE estima, entre otras variables, el número de establecimientos, habitaciones y plazas, así como el número de viajeros, pernoctaciones y grado de ocupación.

Para el mercado p2p de alojamientos turísticos, en cambio, solo existen datos oficiales hace relativamente poco tiempo. Hasta entonces, las investigaciones sobre este mercado se han basado, con frecuencia, en encuestas llevadas a cabo por los propios investigadores o en los datos que proporcionaban iniciativas privadas, como Inside Airbnb (2022), que, mediante técnicas de scraping, recopilaban y ordenaban la información de los anuncios de los alojamientos que las plataformas publican en Internet y la ponían a disposición de los interesados. Aunque esta última opción ha sido habitual para sustentar los análisis (Dann et al., 2019), posee algunas debilidades (Alsudais, 2021) y, en cualquier caso, estaban referidas a ciudades o territorios concretos.

Las bases para un análisis apoyado en datos fiables y exhaustivos sobre el mercado p2p de alojamientos turísticos empezaron a sentarse cuando, en marzo de 2020, la Comisión Europea anunció un acuerdo con Airbnb, Booking, Expedia y Tripadvisor para acceder a información sobre los alojamientos $\triangleright$ 
ofrecidos a través de dichas plataformas (European Commission, 2020). Dicho acuerdo es el que, en última instancia, ha permitido al INE publicar, como estadística experimental, por un lado, la Medición del número de viviendas turísticas en España y su capacidad (INE, 2022a) y, por otro, la Estimación de la ocupación en alojamientos turísticos a partir de datos de plataformas digitales (INE, 2022b). Hay que advertir que la medición del número de viviendas turísticas deja fuera a otros alojamientos turísticos de corta estancia que sí incluye la segunda estadística mencionada. En consecuencia, al referirse a ámbitos poblacionales distintos, ambas estadísticas no pueden tratarse de forma conjunta. A pesar de todo, pueden utilizarse de forma independiente para establecer comparaciones con la Encuesta de ocupación hotelera y, de esta forma, detectar las diferencias que existen entre el mercado hotelero y el de alojamientos p2p, tanto por el lado de la oferta como de la demanda.

\section{Resultados y discusión}

La presentación de los resultados se agrupará en los relacionados con la oferta, referido al número de plazas, y de la demanda, donde se utilizarán las cifras de alojamiento. En ambos casos se dispone de información tanto para el mercado hotelero como para el mercado de alojamientos p2p. Para este último mercado solo se dispone de datos sobre las plazas para algunos meses y sobre los alojamientos para determinados años, y el INE no proporciona información sobre los precios, lo cual, como es obvio, restringe el análisis que cabe realizar cuando se pretende una comparación con el mercado hotelero.

\subsection{Oferta: plazas}

En la Encuesta de ocupación hotelera el número de plazas equivale al número de camas fijas del establecimiento (sin incluir las supletorias), teniendo en cuenta que las de matrimonio dan lugar a dos plazas (INE, 2019). Por su parte, para las viviendas p2p, el número de plazas se contabiliza según la asignación que realiza la plataforma de referencia (INE, 2020). Por tanto, no se trata de mediciones que, en sentido estricto, sean idénticas. No obstante, son una buena aproximación de la capacidad de alojamiento en cada mercado que permite un análisis comparativo.

La primera estimación de las plazas para las viviendas $p 2 p$ publicada por el INE está referida a agosto de 2020 y la más reciente corresponde al mismo mes de 2021. Son las fechas que se han tomado como referencia para elaborar la Tabla 1, que contiene el número de plazas estimadas para los hoteles y las viviendas $p 2 p$, y que sirve de base para conocer lo ocurrido durante la pandemia en ambos mercados. De entrada, contrasta el incremento en el número de plazas estimadas para los hoteles $(36 \%)$ con la caída de las plazas en las viviendas turísticas $(-4 \%)$ en el periodo considerado.

En el caso de los hoteles hay que tener en cuenta que, tras la declaración del estado de alarma el 14 de marzo de 2020 (R.D. 463/2020), se suspendió la apertura al público de los establecimientos de alojamiento turístico mediante Orden SND/257/2020, de 19 de marzo. Posteriormente, cuando se flexibilizaron las medidas, la reapertura de los establecimientos fue parcial. Además, con los rebrotes de las primeras semanas de agosto se reactivaron las restricciones a los viajes internacionales, lo cual condicionó las reaperturas previstas y $\triangleright$ 
TABLA 1

NÚMERO DE PLAZAS ESTIMADAS EN HOTELES Y VIVIENDAS P2P POR COMUNIDADES AUTÓNOMAS

(Agosto 2020-agosto 2021)

\begin{tabular}{|c|c|c|c|c|c|c|}
\hline \multirow[b]{2}{*}{ Comunidades autónomas } & \multicolumn{3}{|c|}{ Hoteles } & \multicolumn{3}{|c|}{ Viviendas p2p } \\
\hline & $\begin{array}{c}2020 \\
\text { (agosto) }\end{array}$ & $\begin{array}{c}2021 \\
\text { (agosto) }\end{array}$ & $\Delta \%$ & $\begin{array}{c}2020 \\
\text { (agosto) }\end{array}$ & $\begin{array}{c}2021 \\
\text { (agosto) }\end{array}$ & $\Delta \%$ \\
\hline Andalucía & 243.542 & 286.233 & 17,5 & 340.256 & 320.089 & $-5,9$ \\
\hline Aragón & 34.069 & 38.551 & 13,2 & 20.167 & 19.339 & $-4,1$ \\
\hline Asturias & 27.552 & 28.686 & 4,1 & 24.698 & 25.851 & 4,7 \\
\hline Baleares & 171.880 & 306.027 & 78,0 & 182.748 & 181.713 & $-0,6$ \\
\hline Canarias & 133.321 & 214.150 & 60,6 & 162.121 & 167.141 & 3,1 \\
\hline Cantabria & 20.488 & 21.902 & 6,9 & 24.578 & 26.980 & 9,8 \\
\hline Castilla y León & 51.304 & 53.059 & 3,4 & 33.058 & 32.713 & $-1,0$ \\
\hline Castilla-La Mancha & 25.879 & 29.555 & 14,2 & 20.317 & 20.288 & $-0,1$ \\
\hline Cataluña & 201.166 & 283.170 & 40,8 & 328.147 & 302.317 & $-7,9$ \\
\hline C. Valenciana & 106.536 & 132.452 & 24,3 & 283.900 & 266.464 & $-6,1$ \\
\hline Extremadura & 16.524 & 17.261 & 4,5 & 6.594 & 6.260 & $-5,1$ \\
\hline Galicia & 66.345 & 70.147 & 5,7 & 67.050 & 72.753 & 8,5 \\
\hline Madrid & 51.483 & 93.364 & 81,3 & 70.522 & 61.127 & $-13,3$ \\
\hline Murcia & 15.455 & 17.190 & 11,2 & 32.079 & 30.112 & $-6,1$ \\
\hline Navarra & 10.825 & 11.389 & 5,2 & 4.906 & 4.663 & $-5,0$ \\
\hline País Vasco & 27.612 & 31.086 & 12,6 & 19.424 & 18.359 & $-5,5$ \\
\hline La Rioja & 5.344 & 6.186 & 15,8 & 6.401 & 6.208 & $-3,0$ \\
\hline Total & 1.209 .325 & 1.640 .408 & 35,6 & 1.626 .966 & 1.562 .377 & $-4,0$ \\
\hline
\end{tabular}

provocó el nuevo cierre de establecimientos (Exceltur, 2021). El resultado fue que, en agosto de 2020 , se estimaron algo más de 13.000 establecimientos hoteleros abiertos, lo que suponía la menor cifra, al menos desde 1999. El año siguiente mejoró la situación, con casi 15.000 establecimientos abiertos, aunque seguía muy alejado de los casi 17.000 que se contabilizaron en agosto de 2019 , el año previo a la pandemia. Estos datos sirven para explicar el crecimiento experimentado en el número de plazas hoteleras estimadas y para subrayar que, en agosto de 2021, todavía no se había recuperado la oferta hotelera que existía antes de la pandemia. En cualquier caso, en todas las comunidades autónomas se registró una subida en el número de las plazas hoteleras entre agosto de 2020 y agosto de 2021. Los incrementos más importantes se dieron en
Baleares (78\%), Canarias $(61 \%)$, Cataluña (41\%) y Madrid ( $81 \%)$.

La oferta de viviendas $p 2 p$ ha tenido una dinámica diferente a la del mercado hotelero. No se dispone de datos previos a la pandemia, pero la Tabla 1 sugiere que muchos propietarios dejaron de ofrecer su vivienda en el mercado p2p. Es decir, mientras que los establecimientos hoteleros cerraron temporalmente en el verano de 2020 y, más tarde, muchos de ellos optaron por la reapertura, los datos apuntan a que un número considerable de propietarios de viviendas $\mathrm{p} 2 \mathrm{p}$ abandonaron definitivamente el mercado durante la pandemia. En efecto, la mayor flexibilidad de la oferta en el mercado p2p de alojamientos turísticos, en relación con la de los hoteles, se explica por el menor peso que tienen los costes fijos en relación con los costes totales, lo cual facilita $\triangleright$ 
tanto la entrada como la salida del mercado (Einav et al., 2016). En este contexto, se ha sugerido que, ante la crisis turística provocada por la pandemia, se ha producido un trasvase de viviendas desde el mercado de alquiler a corto plazo hacia el de alquiler a largo plazo (Dolnicar y Zare, 2020). No obstante, también en este caso, se aprecian notables diferencias entre comunidades autónomas. El mayor descenso, en el periodo considerado, se produjo en Madrid (-13\%), mientras que en la agrupación geográfica que integran las comunidades de Asturias (4,7\%), Cantabria ( $9,8 \%$ ) y Galicia $(8,5 \%)$, y en Canarias $(3,1 \%)$, se registran subidas apreciables.

En el Gráfico 1 se compara, teniendo en cuenta las salvedades señaladas, las plazas en los hoteles y en las viviendas p2p. En términos generales se produce un descenso de la ratio plazas en viviendas $\mathrm{p} 2 \mathrm{p}$ / plazas en hoteles, salvo en Asturias, Cantabria y Galicia, en donde se experimentan ligeras subidas. Otro hecho relevante se refiere a la importancia de la capacidad de alojamiento de las viviendas $p 2 p$, que ha alcanzado en muchas regiones niveles similares 0 , incluso, superiores a la de los hoteles. En este sentido, son reseñables las comunidades peninsulares del arco mediterráneo (Cataluña, C. Valenciana, Murcia y Andalucía). También mantienen una ratio superior a la unidad en agosto de 2021 Cantabria, Galicia y La Rioja.

Ahora bien, aunque el análisis de la evolución de las plazas durante la pandemia tiene interés como indicador de la capacidad potencial de alojamiento, es necesario apuntar $\triangleright$

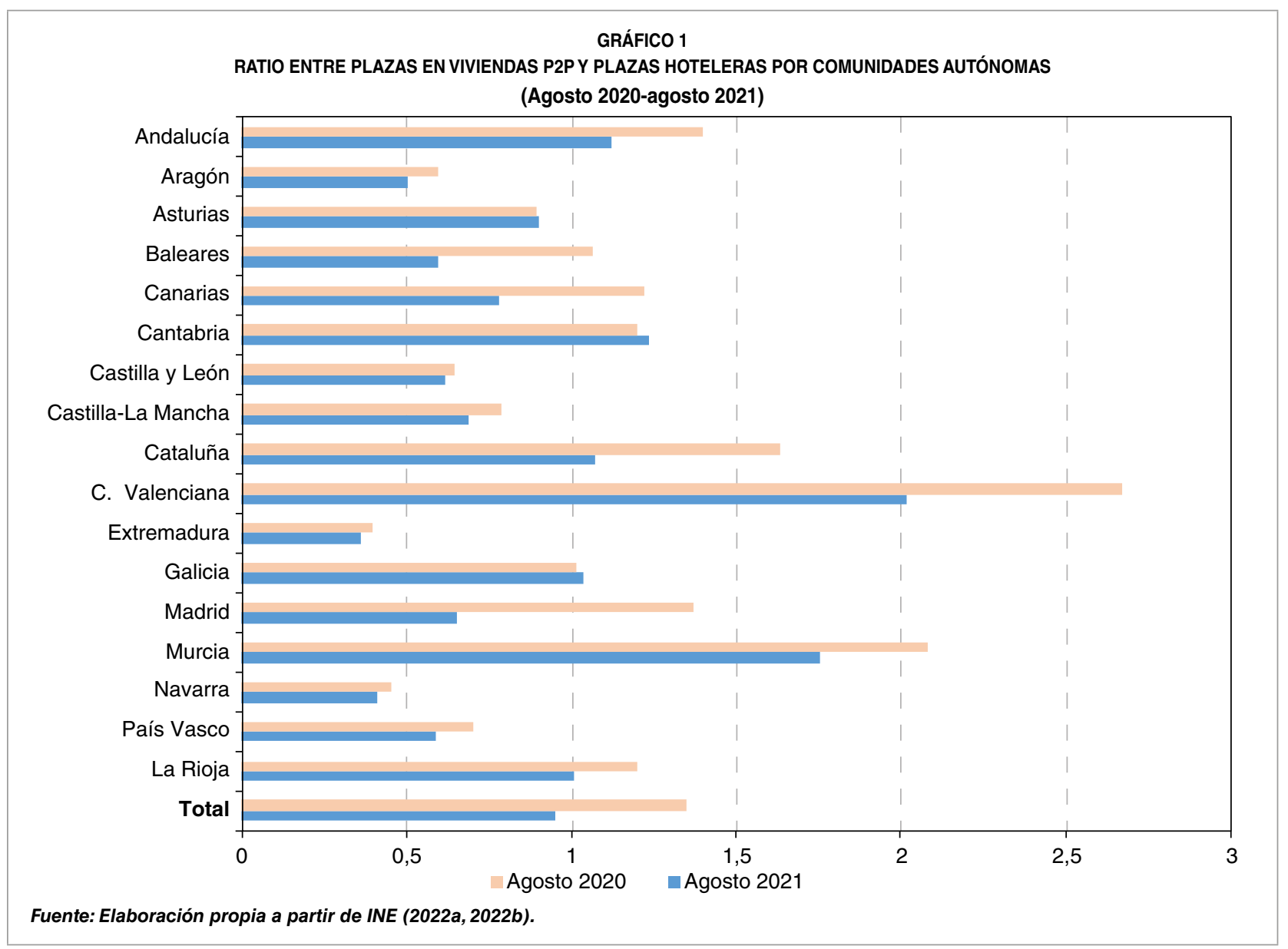


que existe evidencia de que en el mercado $p 2 p$ la mayoría de las viviendas apenas reciben solicitudes de reserva y, en consecuencia, tienen una ocupación escasa o nula, ya que la demanda se suele concentrar en una parte de la oferta (Benítez-Aurioles, 2020b). De hecho, el porcentaje de valoraciones realizadas por los huéspedes (que, frecuentemente se ha utilizado como indicador de la demanda), acumulado por el $20 \%$ de las viviendas p2p que tienen más valoraciones, suele ser superior al $70 \%$ (Benítez-Aurioles, 2021b). Además, debe tenerse en cuenta que, aunque se reduzca la oferta de alojamientos, puede producirse una utilización más intensiva de los que permanecen en el mercado, con lo que, en última instancia, se estaría produciendo un incremento en el número de los que realmente se comercializan. En consecuencia, es preciso acudir a algún indicador de la demanda turística o de la ocupación efectiva para tener una visión más precisa de los mercados que estamos considerando. Para ello utilizaremos la evolución de las pernoctaciones.

\subsection{Demanda: pernoctaciones}

Según la Encuesta de ocupación hotelera, la pernoctación equivale a la plaza ocupada cada noche por un viajero que se aloja en un establecimiento (INE, 2019). De manera similar, el proyecto técnico de Estimación de la ocupación en alojamientos turísticos y otros alojamientos de corta estancia considera que las pernoctaciones son las plazas ocupadas cada noche que un viajero utiliza en un alojamiento. Para que no haya dudas, se aclara lo siguiente: «Una familia de 4 miembros que se aloja durante 3 noches en un apartamento, da lugar a 1 alojamiento turístico ocupado, 3 noches ocupadas y 12 pernoctaciones» (INE, 2021). No obstante, hay que advertir que dicha estimación se refiere al grupo completo 55.2 de la Clasificación Nacional de Actividades Económicas (CNAE), mientras que los datos referidos a la medición del número de viviendas turísticas, analizados en el apartado anterior, excluye «otros alojamientos de corta estancia». Esta es la razón por la que, en este apartado, utilizamos la expresión «alojamiento p2p» y no «vivienda p2p», como hicimos anteriormente.

Para las pernoctaciones en alojamientos turísticos y otros alojamientos de corta estancia sí se dispone de datos para los años previos a la pandemia. En este sentido, los datos recogidos en el Gráfico 2 son muy expresivos del efecto que, a nivel agregado, ha tenido la pandemia. Mientras que en 2019 las pernoctaciones totales crecieron un $3 \%$ respecto a 2018 , hasta alcanzar 449 millones, en 2020 se redujeron, respecto al año anterior, en un $70 \%$ al registrarse algo más de 136 millones. Además, el diferente comportamiento que tuvieron las pernoctaciones en los hoteles respecto a las de los alojamientos $p 2 p$ pone de manifiesto la creciente importancia de estas últimas. En efecto, en 2019, el año previo a la pandemia, el número de pernoctaciones en los alojamientos p2p experimentó un crecimiento relativo muy superior $(11,9 \%)$ al de los hoteles, que parecían estancarse (0,9\%). En 2020, el primer año de la pandemia, se contabilizó un descenso relativo menor en las pernoctaciones en los alojamientos p2p $(-58,1 \%)$ que en los hoteles $(-73,1 \%)$. En consecuencia, al crecer más en 2019 y decrecer menos en 2020 , el porcentaje que representaron las pernoctaciones en los alojamientos p2p sobre el total pasó de un $22 \%$ en 2018 al $32 \%$ en 2020 . Estos datos avalan la relevancia que ha alcanzado el mercado $p 2 p$ en relación con el mercado convencional. 


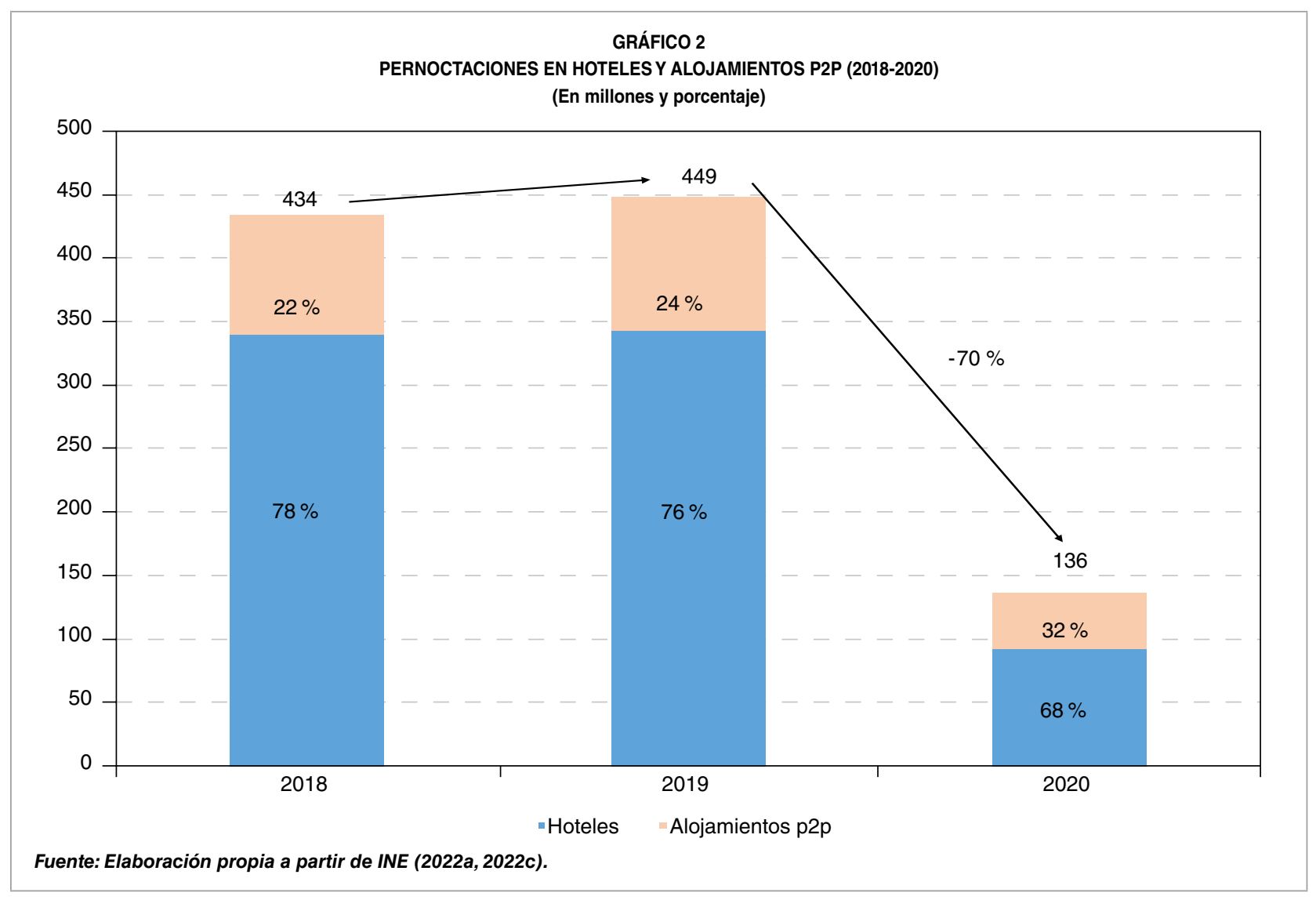

En la Tabla 2 se observa cómo se reproducen por comunidades autónomas las tendencias señaladas a nivel agregado: ligero crecimiento de las pernoctaciones hoteleras en 2019 respecto a 2018 que, incluso, en Baleares, Canarias y La Rioja se convierten en decrementos; fuerte aumento en el mercado de alojamientos p2p, aunque mucho más moderado en los archipiélagos y Cataluña; y, en 2020, tasas de decremento menores en las pernoctaciones en los alojamientos $\mathrm{p} 2 \mathrm{p}$ respecto a los hoteles en todas las comunidades autónomas.

En el Gráfico 3 se ha representado la evolución mensual de las pernoctaciones. En los años previos a la pandemia se observa el componente estacional de las series, que alcanza su máximo en agosto, la preponderancia de las pernoctaciones en los hoteles frente a las de los alojamientos p2p, y la ligera aproximación de estas últimas a las primeras en 2019. Asimismo, el colapso del mercado, a raíz de la declaración del estado de alarma en marzo de 2020, se manifiesta en la práctica ausencia de actividad hasta junio. Es interesante mencionar que, precisamente, en abril, mayo y junio las pernoctaciones en los alojamientos p2p superan, por primera vez, según la información que estamos manejando, a las de los hoteles, aunque levemente. En este sentido, es posible que, ante el cierre de los hoteles, los viajeros buscaran una alternativa en el mercado $p 2 p$ para garantizar su estancia en los lugares de destino, lo que explicaría que, en el conjunto de los tres meses señalados, las pernoctaciones registradas en los alojamientos p2p (2,3 millones) fueran más numerosas que las de los hoteles (2,1 millones).

El levantamiento de algunas restricciones propició una cierta recuperación de la $\triangleright$ 
EL IMPACTO DE LA PANDEMIA EN EL MERCADO DE ALOJAMIENTOS TURÍSTICOS EN ESPAÑA...

TABLA 2

NÚMERO DE PERNOCTACIONES (EN MILLONES) E INCREMENTO PORCENTUAL RESPECTO AL AÑO ANTERIOR ( $\%$ \%)

POR COMUNIDADES AUTÓNOMAS

(Hoteles y alojamientos p2p, 2019 y 2020)

\begin{tabular}{|c|c|c|c|c|c|c|c|c|}
\hline \multirow{3}{*}{ Comunidad autónoma } & \multicolumn{4}{|c|}{ Hoteles } & \multicolumn{4}{|c|}{ Alojamientos p2p } \\
\hline & \multicolumn{2}{|c|}{2019} & \multicolumn{2}{|c|}{2020} & \multicolumn{2}{|c|}{2019} & \multicolumn{2}{|c|}{2020} \\
\hline & Núm. & $\Delta \%$ & Núm. & $\Delta \%$ & Núm. & $\Delta \%$ & Núm. & $\Delta \%$ \\
\hline Andalucía & 54,8 & 2,9 & 16,8 & $-69,3$ & 24,9 & 18,2 & 10,3 & $-58,8$ \\
\hline Aragón & 5,8 & 1,9 & 2,6 & $-55,2$ & 1,2 & 20,8 & 0,7 & $-38,4$ \\
\hline Asturias & 3,7 & 4,5 & 1,9 & $-50,5$ & 1,3 & 28,3 & 0,9 & $-30,7$ \\
\hline Baleares & 58,1 & $-1,8$ & 6,0 & $-89,7$ & 8,4 & 3,8 & 2,6 & $-68,8$ \\
\hline Canarias & 67,3 & $-2,5$ & 20,1 & $-70,2$ & 14,9 & 4,1 & 8,0 & $-46,4$ \\
\hline Cantabria & 2,9 & 0,9 & 1,4 & $-53,3$ & 1,2 & 19,0 & 0,8 & $-33,8$ \\
\hline Castilla y León & 8,5 & 3,8 & 3,2 & $-62,5$ & 1,4 & 33,8 & 0,7 & $-48,3$ \\
\hline Castilla-La Mancha & 3,8 & 1,1 & 1,6 & $-58,8$ & 0,8 & 32,9 & 0,4 & $-47,9$ \\
\hline Cataluña & 58,2 & 2,6 & 13,3 & $-77,1$ & 20,7 & 6,4 & 7,1 & $-65,9$ \\
\hline C. Valenciana & 29,8 & 0,8 & 8,7 & $-70,7$ & 15,8 & 12,5 & 6,7 & $-57,8$ \\
\hline Extremadura & 2,5 & 1,5 & 1,1 & $-58,3$ & 0,4 & 21,5 & 0,2 & $-44,1$ \\
\hline Galicia & 9,0 & 5,9 & 3,5 & $-60,8$ & 2,4 & 39,7 & 1,4 & $-40,1$ \\
\hline Madrid & 25,7 & 4,6 & 7,4 & $-71,0$ & 8,2 & 13,3 & 2,7 & $-67,6$ \\
\hline Murcia & 3,3 & 0,0 & 1,1 & $-65,8$ & 1,3 & 12,7 & 0,6 & $-53,1$ \\
\hline Navarra & 2,0 & 3,0 & 0,7 & $-63,1$ & 0,4 & 27,1 & 0,2 & $-52,8$ \\
\hline País Vasco & 6,2 & 2,4 & 2,4 & $-61,2$ & 1,7 & 13,4 & 0,7 & $-60,9$ \\
\hline La Rioja & 1,0 & $-0,8$ & 0,3 & $-65,1$ & 0,4 & 28,4 & 0,2 & $-57,7$ \\
\hline Total & 342,7 & 0,9 & 92,1 & $-73,1$ & 105,6 & 11,9 & 44,2 & $-58,1$ \\
\hline
\end{tabular}

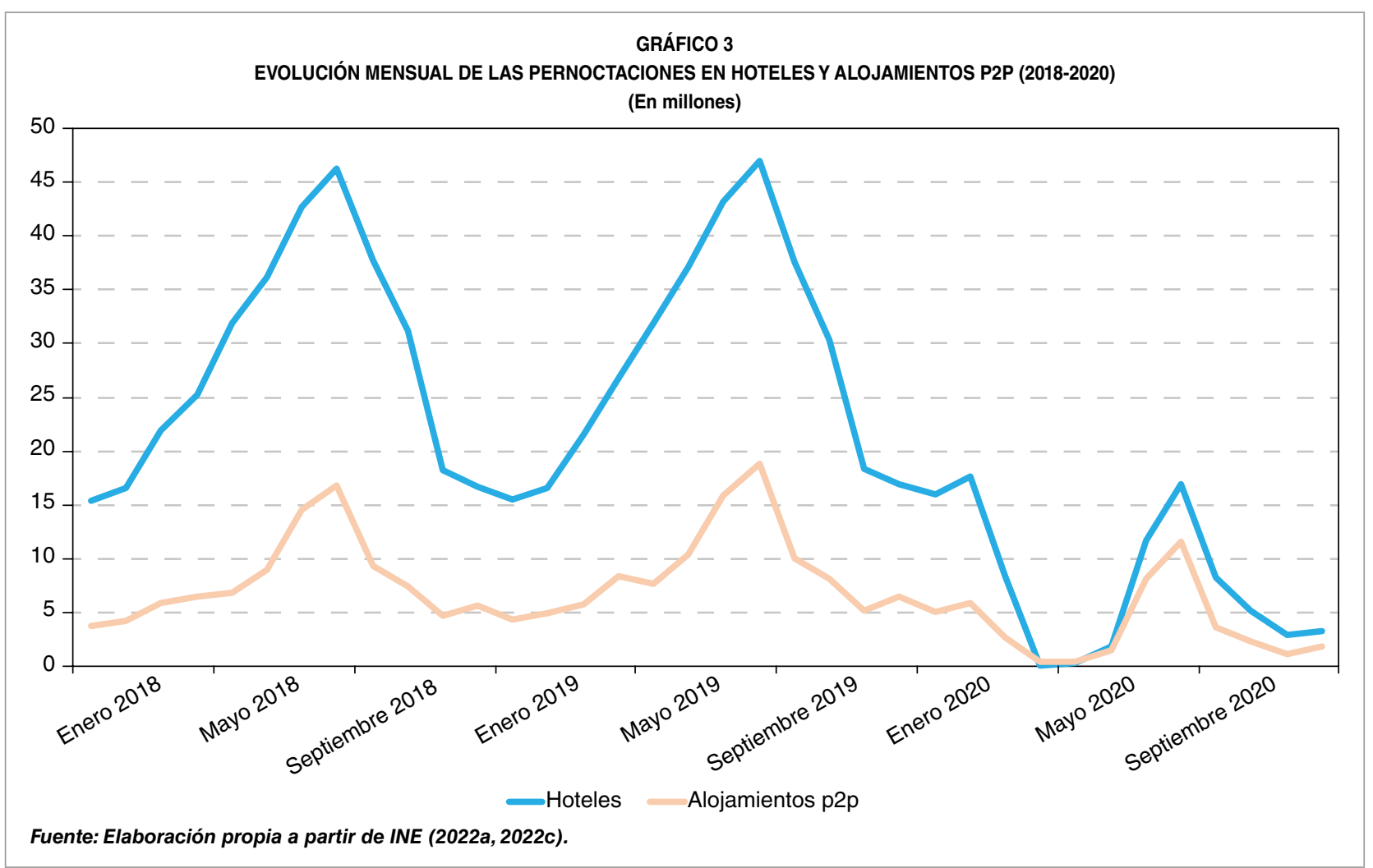


TABLA 3

NÚMERO DE PERNOCTACIONES (EN MILLONES) E INCREMENTO PORCENTUAL RESPECTO AL AÑO ANTERIOR $(\Delta \%)$ DE LOS RESIDENTES EN ESPAÑA Y DE LOS NO RESIDENTES

\begin{tabular}{|c|c|c|c|c|c|c|c|c|}
\hline & \multicolumn{4}{|c|}{ Hoteles } & \multicolumn{4}{|c|}{ Alojamientos p2p } \\
\hline & \multicolumn{2}{|c|}{2019} & \multicolumn{2}{|c|}{2020} & \multicolumn{2}{|c|}{2019} & \multicolumn{2}{|c|}{2020} \\
\hline & Núm. & $\Delta \%$ & Núm. & $\Delta \%$ & Núm. & $\Delta \%$ & Núm. & $\Delta \%$ \\
\hline Residentes & 119,6 & 2,7 & 51,2 & $-57,2$ & 34,3 & 18,1 & 24,5 & $-28,7$ \\
\hline No residentes & 223,4 & 0,0 & 41,1 & $-81,6$ & 71,3 & 9,1 & 19,7 & $-72,3$ \\
\hline Total & 343,0 & 0,9 & 92,2 & $-73,1$ & 105,6 & 11,9 & 44,2 & $-58,1$ \\
\hline
\end{tabular}

actividad turística a partir de junio de 2020 y el aumento en el número de pernoctaciones en los hoteles que recuperan el protagonismo, aunque con cifras similares a las registradas en los alojamientos p2p. No obstante, ante la aparición de nuevos rebrotes y medidas restrictivas, en los últimos meses del año la actividad turística fue muy escasa (Exceltur, 2021).

Una desagregación según el lugar de procedencia de los viajeros proporciona información interesante sobre la evolución reciente del mercado de alojamientos convencionales respecto al mercado p2p. Tal como se recoge en la Tabla 3, en 2019 aparecen algunas señales que apuntan hacia cierto estancamiento en las pernoctaciones de los residentes en el extranjero en los hoteles que contrastan con la dinámica que manifiestan en el mercado p2p. En 2019 el número de pernoctaciones de los extranjeros en los hoteles apenas se modifican respecto a las cifras de 2018, mientras que crecen en más de un $9 \%$ las que realizan en los alojamientos p2p. También los residentes en España siguen la misma pauta con incrementos moderados en $2019(2,7 \%)$ en las pernoctaciones en los hoteles, y subidas destacables en las pernoctaciones en los alojamientos p2p (18,1\%). Es decir, antes de la pandemia ya se apreciaban indicios del mayor dinamismo del mercado $p 2 p$ respecto al mercado convencional. En este sentido, el shock que provocó la pandemia en el sector turístico puede que, simplemente, haya acelerado ese proceso. Así, los datos de 2020, tal como se ha apuntado más arriba, registran descensos más intensos en el número de las pernoctaciones en los hoteles que, según la Tabla 3, se explica en mayor medida por el comportamiento de los no residentes. En este punto, conviene señalar que el descenso menos intenso de las pernoctaciones de los residentes podría explicarse, no solo por las restricciones que han tenido los extranjeros para hacer turismo en España, sino también por la sustitución, por parte de los españoles, de los viajes al extranjero por los realizados en el interior del país como consecuencia de la pandemia (Benítez-Aurioles, 2021c).

De acuerdo con lo expuesto parece pertinente plantear la hipótesis de que, una vez superada la pandemia, se refuerce la orientación de la demanda hacia el mercado p2p de alojamientos turísticos.

\section{Conclusiones}

La pandemia desencadenada a principios de 2020 ha afectado especialmente al turismo, aunque con un impacto sectorial y territorial heterogéneo. Particularmente, en el caso de los alojamientos turísticos resulta pertinente distinguir los hoteles de los alojamientos que se comercializan en el mercado p2p. Así, gracias a la reciente publicación por parte del $\triangleright$ 
INE de estadísticas que ofrecen datos fiables, tanto por el lado de la oferta como de la demanda, del mercado $\mathrm{p} 2 \mathrm{p}$, se han identificado algunos rasgos diferenciales.

Por el lado de la oferta se ha constatado que, después de la suspensión de la apertura al público de los establecimientos de alojamiento turístico, como consecuencia del estado de alarma, y de la drástica caída de la demanda turística, muchos hoteles permanecieron cerrados en el verano de 2020. Al año siguiente, cuando se flexibilizaron las medidas y hubo una cierta recuperación de la demanda, se produjo una reapertura de establecimientos que, sin alcanzar los niveles previos a la pandemia, fue generalizada en todas las comunidades autónomas. En cambio, en el mercado p2p existen indicios de que muchos propietarios dejaron de ofrecer sus viviendas y abandonaron definitivamente el mercado, quizás, para orientar sus propiedades hacia el mercado de alquiler a largo plazo. No obstante, aunque la disminución de la oferta de viviendas $p 2 p$, entre agosto de 2020 y agosto de 2021, fue evidente a nivel general, en algunas comunidades autónomas (Asturias, Cantabria, Galicia y Canarias) se registraron subidas significativas. En cualquier caso, los últimos datos disponibles demuestran que la capacidad de alojamiento del mercado p2p es muy considerable y que, en algunas regiones, sigue teniendo una capacidad de alojamiento superior a la que proporciona el mercado convencional.

Por el lado de la demanda, las cifras de pernoctaciones antes de la pandemia, dentro de la preponderancia que tenía el mercado hotelero, indicaban el mayor dinamismo que estaba experimentando el mercado p2p debido, especialmente, al comportamiento de los residentes en el extranjero. Además, durante la pandemia se registró un descenso menos intenso en las pernoctaciones en el mercado $p 2 p$ respecto a los hoteles, lo que, en consecuencia, parecía estar reforzando la cuota de mercado de los alojamientos p2p respecto a los alojamientos convencionales. En cualquier caso, una vez superada la pandemia, y se disponga de la información estadística necesaria, se podrá verificar si se consolidan las tendencias que hemos señalado y, en particular, la creciente orientación de la demanda turística hacia el mercado p2p.

\section{Bibliografía}

Alsudais, A. (2021). Incorrect data in the widely used Inside Airbnb dataset. Decision Support Systems, 141. https://doi.org/10.1016/j.dss.2020. 113453

Bae, S. Y., \& Chang, P. J. (2021). The effect of coronavirus disease-19 (COVID-19) risk perception on behavioural intention towards 'untact' tourism in South Korea during the first wave of the pandemic. Current Issues in Tourism, 24(7), 10171035. https://doi.org/10.1080/13683500.2020.17 98895

Banco de España. (2021). Informe Anual 2020. https://www.bde.es/f/webbde/SES/Secciones/ Publicaciones/PublicacionesAnuales/InformesAnuales/20/Fich/InfAnual_2020.pdf

Bausch, T., Gartner, W. C., \& Ortanderl, F. (2021). How to Avoid a COVID-19 Research Paper Tsunami? A Tourism System Approach. Journal of Travel Research, 60(3), 467-485. https://doi. org/10.1177/0047287520972805

Benítez-Aurioles, B. (2019). Is Airbnb bad for hotels? Current Issues in Tourism, 1-4. https://doi.org/10. 1080/13683500.2019.1646226

Benítez-Aurioles, B. (2020a). Peer-to-peer market for tourist accommodation: characterization and implications. UMA Editorial. https://hdl.handle. net/10630/20842 
Benítez-Aurioles, B. (2020b). The exhaustion of the herding effect in peer-to-peer accommodation. Tourism Economics. https://doi.org/10.1177/ 1354816620946537

Benítez-Aurioles, B. (2021a). How the peer-to-peer market for tourist accommodation has responded to COVID-19. International Journal of Tourism Cities. https://doi.org/10.1108/IJTC-07-2021-0140

Benítez-Aurioles, B. (2021b). A proposal to regulate the peer-to-peer market for tourist accommodation. International Journal of Tourism Research, 23(1), 70-78. https://doi.org/10.1002/ jtr.2393

Benítez-Aurioles, B. (2021c). El turismo interior en España ante la crisis de la COVID-19. Boletín Económico de Información Comercial Española, (3139), 43-53. https://doi.org/10.32796/bice. 2021.3139 .7285

Blal, I., Singal, M., \& Templin, J. (2018). Airbnb's effect on hotel sales growth. International Journal of Hospitality Management, 73, 85-92. https:// doi.org/10.1016/j.ijhm.2018.02.006

Bresciani, S., Ferraris, A., Santoro, G., Premazzi, K., Quaglia, R., Yahiaoui, D., \& Viglia, G. (2021). The seven lives of Airbnb. The role of accommodation types. Annals of Tourism Research, 88. https:// doi.org/10.1016/j.annals.2021.103170

Choi, K. H., Jung, J., Ryu, S., Kim, S. D., \& Yoon, S. M. (2015). The Relationship between Airbnb and the Hotel Revenue: In the case of Korea. Indian Journal of Science and Technology, 8(26), 1- 8. https://doi.org/10.17485/ijst/2015/v8i26/81013

Collins-Kreiner, N., \& Ram, Y. (2021). National tourism strategies during the Covid-19 pandemic. Annals of Tourism Research, 89. https://doi. org/10.1016/j.annals.2020.103076

Dann, D., Teubner, T., \& Weinhardt, C. (2019). Poster child and guinea pig-insights from a structured literature review on Airbnb. International Journal of Contemporary Hospitality Management, 31(1), 427-473.https://doi.org/10.1108/IJCHM-032018-0186
Dogru, T., Hanks, L., Mody, M., Suess, C., \& SirakayaTurk, E. (2020). The effects of Airbnb on hotel performance: Evidence from cities beyond the United States. Tourism Management, 79. https:// doi.org/10.1016/j.tourman.2020.104090

Dogru, T., Mody, M., Suess, C., Line, N., \& Bonn, M. (2020). Airbnb 2.0: Is it a sharing economy platform or a lodging corporation? Tourism Management, 78. https://doi.org/10.1016/j.tourman.2019. 104049

Dolnicar, S., \& Zare, S. (2020). COVID19 and AirbnbDisrupting the disruptor. Annals of Tourism Research, 83. https://doi.org/10.1016/j.annals. 2020.102961

Duro, J. A., Perez-Laborda, A., Turrion-Prats, J., \& Fernández-Fernández, M. (2021). Covid-19 and tourism vulnerability. Tourism Management Perspectives, 38. https://doi.org/10.1016/j.tmp. 2021.100819

Einav, L., Farronato, C., \& Levin, J. (2016). Peerto-Peer Markets. Annual Review of Economics, 8, 615-635. https://doi.org/10.1146/annureveconomics-080315-015334

European Commission. (2020). Commission reaches agreement with collaborative economy platforms to publish key data on tourism accommodation. https://ec.europa.eu/commission/presscorner/ detail/en/ip_20_194

Exceltur. (2021). Barómetro de la rentabilidad de los destinos turísticos españoles. Balance 2020. https://www.exceltur.org/wp-content/uploads/ 2021/02/Baro\%CC\%81metro-Rentabilidad-yel-Empleo-Enero-Diciembre-2020.pdf

Farzanegan, M. R., Gholipour, H. F., Feizi, M., Nunkoo, R., \& Andargoli, A. E. (2021). International Tourism and Outbreak of Coronavirus (COVID-19): A Cross-Country Analysis. Journal of Travel Research, 60(3), 687-692. https://doi. org/10.1177/0047287520931593

Gretzel, U., Fuchs, M., Baggio, R., Hoepken, W., Law, R., Neidhardt, J., Pesonen, J., Zanker, M., \& Xiang, Z. (2020). e-Tourism beyond $D$ 


\section{EL IMPACTO DE LA PANDEMIA EN EL MERCADO DE ALOJAMIENTOS TURÍSTICOS EN ESPAÑA...}

COVID-19: a call for transformative research. Information Technology \& Tourism, 22, 187-203. https://doi.org/10.1007/s40558-020-00181-3

Guttentag, D. (2015). Airbnb: disruptive innovation and the rise of an informal tourism accommodation sector. Current issues in Tourism, 18(12), 1192-1217. https://doi.org/10.1080/13683500.201 3.827159

Gyódi, K. (2021). Airbnb and hotels during COVID-19: different strategies to survive. International Journal of Culture, Tourism and Hospitality Research. https://doi.org/10.1108/IJCTHR-09-2020-0221

Hartmans, A. (2017, August 10). Airbnb now has more listings worldwide than the top five hotel brands combined. Insider. https://www.businessinsider.com/airbnb-total-worldwide-listings2017-8

Heo, C. Y., Blal, I., \& Choi, M. (2019). What is happening in Paris? Airbnb, hotels, and the Parisian market: A case study. Tourism Management, 70, 7888. https://doi.org/10.1016/j.tourman.2018.04.003

Higgins-Desbiolles, F. (2021). The «war over tourism»: challenges to sustainable tourism in the tourism academy after COVID-19. Journal of Sustainable Tourism, 29(4), 551-569. https://doi. org/10.1080/09669582.2020.1803334

Hu, X., Yan, H., Casey, T., \& Wu, C. H. (2021). Creating a safe haven during the crisis: How organizations can achieve deep compliance with COVID-19 safety measures in the hospitality industry. International Journal of Hospitality Management, 92. https://doi.org/10.1016/j.ijhm.2020. 102662

Huang, A., Makridis, C., Baker, M., Medeiros, M., \& Guo, Z. (2020). Understanding the impact of COVID-19 intervention policies on the hospitality labor market. International Journal of Hospitality Management, 91. https://doi.org/10.1016/j. ijhm.2020.102660

INE. (2019). Encuesta de ocupación hotelera. Metodología. https://ine.es/daco/daco42/ocuphotel/ meto_eoh.pdf
INE. (2020). Medición del número de viviendas turísticas en España y su capacidad. Proyecto técnico. https://ine.es/experimental/viv_turistica/ exp_viv_turistica_proyecto.pdf

INE. (2021). Estimación de la ocupación en alojamientos turísticos y otros alojamientos de corta estancia (grupo 55.2 de la CNAE-2009) a partir de información suministrada por plataformas digitales. Proyecto técnico. https://ine.es/experimental/ocupacion/exp_ocupacion_plataforma.pdf

INE. (2022a). Encuesta de ocupación hotelera. https://ine.es/dynt3/inebase/index.htm?padre=239

INE. (2022b). Medición del número de viviendas turísticas en España y su capacidad. https://ine. es/experimental/viv_turistica/experimental_viv_ turistica.htm

INE. (2022c). Estimación de la ocupación en alojamientos turísticos a partir de datos de plataformas digitales. https://ine.es/experimental/ocupacion/experimental_ocupacion.htm

Inside Airbnb. (2022). Get data. http://insideairbnb. com/get-the-data.html

Jang, S., \& Kim, J. (2022). Remedying Airbnb COVID-19 disruption through tourism clusters and community resilience. Journal of Business Research, 139, 529-542. https://doi.org/10.1016/j. jbusres.2021.10.015

Jiménez, J. L., Ortuño, A., \& Pérez-Rodríguez, J. V. (2021). How does AirBnb affect local Spanish tourism markets? Empirical Economics, 1-31. https://doi.org/10.1007/s00181-021-02107-2

Kaczmarek, T., Perez, K., Demir, E., \& Zaremba, A. (2021). How to survive a pandemic: The corporate resiliency of travel and leisure companies to the COVID-19 outbreak. Tourism Management, 84. https://doi.org/10.1016/j.tourman.2020. 104281

Khan, A., Bibi, S., Lyu, J., Latif, A., \& Lorenzo, A. (2021). COVID-19 and sectoral employment trends: assessing resilience in the US leisure and hospitality industry. Current Issues in $D$ 
Tourism, 24(7), 952-969. https://doi.org/10.1080/ 13683500.2020 .1850653

Lai, I. K. W., \& Wong, J. W. C. (2020). Comparing crisis management practices in the hotel industry between initial and pandemic stages of COVID-19. International Journal of Contemporary Hospitality Management, 32(10), 3135-3156. https://doi.org/10.1108/IJCHM-04-2020-0325

Lee, C. C., \& Chen, M. P. (2020). The impact of COVID-19 on the travel and leisure industry returns: Some international evidence. Tourism Economics. https://doi.org/10.1177/1354816620971981

Llaneza Hesse, C., \& Raya Vilchez, J. M. (2021). The effect of COVID-19 on the peer-to-peer rental market. Tourism Economics. https://doi. org/10.1177/13548166211044229

Nhamo, G., Dube, K., \& Chikodzi, D. (2020). Counting the Cost of COVID-19 on the Global Tourism Industry. Springer. https://doi.org/10.1007/978-3030-56231-1

Qiu, R. T. R., Park, J., Li, S., \& Song, H. (2020). Social costs of tourism during the COVID-19 pandemic. Annals of Tourism Research, 84. https:// doi.org/10.1016/j.annals.2020.102994

Rachmawati, I., \& Shishido, K. (2020). Travelers' motivations to travel abroad during COVID 19 outbreak. International Journal of Applied Sciences in Tourism and Events, 4(1), 1-11. http://repo.stkippgri-bkl.ac.id/id/eprint/855

Sharma, A., \& Nicolau, J. L. (2020). An open market valuation of the effects of COVID-19 on the travel and tourism industry. Annals of Tourism Research, 83. https://doi.org/10.1016/j.annals.2020.102990

Sharma, G. D., Thomas, A., \& Paul, J. (2021). Reviving tourism industry post-COVID-19: A resiliencebased framework. Tourism Management Perspectives, 37. https://doi.org/10.1016/j.tmp.2020.100786
Škare, M., Ribeiro-Soriano, D. E., \& Porada-Rochoń, M. (2021). Impact of COVID-19 on the travel and tourism industry. Technological Forecasting and Social Change, 163. https://doi.org/10.1016/j. techfore.2020.120469

Strømmen-Bakhtiar, A., \& Vinogradov, E. (2019). The effects of Airbnb on hotels in Norway. Society and Economy, 41(1), 87-105. https://doi. org/10.1556/204.2018.001

Tran, B. L., Chen, C. C., Tseng, W. C., \& Liao, S. Y. (2020). Tourism under the Early Phase of COVID-19 in Four APEC Economies: An Estimation with Special Focus on SARS Experiences. International Journal of Environmental Research and Public Health, 17(20). https://doi. org/10.3390/ijerph17207543

Tse, S., \& Tung, V. W. S. (2021). Residents' discrimination against tourists. Annals of Tourism Research, 88. https://doi.org/10.1016/j.annals.2020. 103060

UNWTO. (2019). Definiciones de turismo de la OMT. https://doi.org/10.18111/9789284420858

Xie, K. L., \& Kwok, L. (2017). The effects of Airbnb's price positioning on hotel performance. International Journal of Hospitality Management, 67, 174-184. https://doi.org/10.1016/j.ijhm.2017. 08.011

Yang, Y., Zhang, C. X., \& Rickly, J. M. (2021). A review of early COVID-19 research in tourism: Launching the Annals of Tourism Research's Curated Collection on coronavirus and tourism. Annals of Tourism Research, 91. https://doi.org/10.1016/j.annals.2021.103313

Zervas, G., Proserpio, D., \& Byers, J. W. (2017). The rise of the sharing economy: Estimating the impact of Airbnb on the hotel industry. Journal of Marketing Research, 54(5), 687-705. https://doi. org/10.1509/jmr.15.0204 